\title{
Global Existence of Weak Solutions to a Fractional Model in Magnetoelastic Interactions
}

\author{
Idriss Ellahiani, ${ }^{1}$ EL-Hassan Essoufi, ${ }^{1}$ and Mouhcine Tilioua ${ }^{2}$ \\ ${ }^{1}$ Laboratoire MISI, FST Settat, Université Hassan I, 26000 Settat, Morocco \\ ${ }^{2}$ Laboratoire M2I, FST Errachidia, Equipe MAMCS, Université Moulay Ismaïl, BP 509, Boutalamine, 52000 Errachidia, Morocco
}

Correspondence should be addressed to Mouhcine Tilioua; tilioua@melix.org

Received 6 July 2016; Accepted 8 September 2016

Academic Editor: Cemil Tunç

Copyright (C) 2016 Idriss Ellahiani et al. This is an open access article distributed under the Creative Commons Attribution License, which permits unrestricted use, distribution, and reproduction in any medium, provided the original work is properly cited.

\begin{abstract}
The paper deals with global existence of weak solutions to a one-dimensional mathematical model describing magnetoelastic interactions. The model is described by a fractional Landau-Lifshitz-Gilbert equation for the magnetization field coupled to an evolution equation for the displacement. We prove global existence by using Faedo-Galerkin/penalty method. Some commutator estimates are used to prove the convergence of nonlinear terms.
\end{abstract}

\section{Introduction}

The nonlinear parabolic hyperbolic coupled system describing magnetoelastic dynamics in $Q=(0, T) \times \Omega(T>0$ and $\Omega$ is a bounded open set of $\mathbb{R}^{d}, d \geqslant 1$ ) is given by (see [1])

$$
\begin{aligned}
\gamma^{-1} \mathbf{m}_{t} & =-\mathbf{m} \times\left(\mathbf{H}_{\mathrm{eff}}+\mathbf{m}_{t}\right) . \\
\rho \mathbf{u}_{t t}-\operatorname{div}\left(\mathcal{S}(\mathbf{u})+\frac{1}{2} \mathscr{L}(\mathbf{m})\right) & =0 .
\end{aligned}
$$

Equation (1), well known in the literature, is the LandauLifshitz-Gilbert (LLG) equation. The unknown $\mathbf{m}$, the magnetization vector, is a map from $\Omega$ to $S^{2}$ (the unit sphere of $\mathbb{R}^{3}$ ) and $\mathbf{m}_{t}$ is its derivative with respect to time. The symbol $\times$ denotes the vector cross product in $\mathbb{R}^{3}$. Moreover we denote by $m_{i}, i=1,2,3$, the components of $\mathbf{m}$. The constant $\gamma$ represents the damping parameter. $\mathbf{H}_{\text {eff }}$ represents the effective field which is given by

$$
\mathbf{H}_{\mathrm{eff}}=a \Delta \mathbf{m}+\boldsymbol{\ell}(\mathbf{m}, \mathbf{u}),
$$

where $a$ is a positive constant and the components of the vector $\boldsymbol{\ell}(\mathbf{m}, \mathbf{u})$ are given by

$$
\ell_{i}=\lambda_{i j k l} m_{j} \epsilon_{k l}(\mathbf{u}) .
$$

Here $\epsilon_{i j}(\mathbf{u})=(1 / 2)\left(\partial_{i} u_{j}+\partial_{j} u_{i}\right)$ stand for the components of the linearized strain tensor $\epsilon, \lambda_{i j k l}=\lambda_{1} \delta_{i j k l}+\lambda_{2} \delta_{i j} \delta_{k l}+$ $\lambda_{3}\left(\delta_{i k} \delta_{j l}+\delta_{i l} \delta_{j k}\right)$ with $\delta_{i j k l}=1$ if $i=j=k=l$ and $\delta_{i j k l}=0$ otherwise.

Equation (2) describes the evolution of the displacement $\mathbf{u}, \rho$ is a positive constant, and the tensors $\mathcal{S}(\mathbf{u}), \mathscr{L}(\mathbf{m})$ are given by

$$
\begin{aligned}
& \mathcal{S}_{k l}=\sigma_{i j k l} \epsilon_{i j}(\mathbf{u}), \\
& \mathscr{L}_{k l}=\lambda_{i j k l} m_{i} m_{j} .
\end{aligned}
$$

$\sigma=\left(\sigma_{i j k l}\right)$ is the elasticity tensor satisfying the following symmetry property:

$$
\sigma_{i j k l}=\sigma_{k l i j}=\sigma_{j i k l} .
$$

Many studies have been done on the fractional LandauLifshitz equation; we quote here, for example, [2], where the existence of weak solutions under periodical boundary condition has been proven for equation of a reduced model for thin-film micromagnetics. In [3], the main purpose is to consider the well-posedness of the fractional Landau-Lifshitz equation without Gilbert damping. The global existence of weak solutions is proved by vanishing viscosity method. 
Note that the existence and asymptotic behaviors of global weak solutions to the one-dimensional periodical fractional Landau-Lifshitz equation modeling the soft micromagnetic materials are studied in [4]. For the magnetoelasticity coupling, in [1], the authors study the three-dimensional case and establish the existence of weak solutions taking into account three terms of the total free energy. Existence and uniqueness of solutions have been proven in [5] for a simplified model and in [6] a one-dimensional penalty problem is discussed and the gradient flow of the associated type Ginzburg-Landau functional is studied. More precisely the authors prove the existence and uniqueness of a classical solution which tends asymptotically for subsequences to a stationary point of the energy functional. Our aim here is to study the coupled system of magnetoelastic interactions with fractional LLG equation.

The rest of the paper is divided as follows. In the next section we present the model equation we will be interested in. Section 3 recalls some useful lemmas. Finally in Section 4 we prove a global existence result of weak solutions to the considered model.

\section{The Model and Main Result}

We assume that $\Omega$ is a subset of $\mathbb{R}$ and the displacement is only in one direction. Specifically, we consider a simple variable space $x$ and assume that $\Omega=(0,2 \pi)$. We take the following system:

$$
\begin{aligned}
\gamma^{-1} \mathbf{m}_{t} & =-\mathbf{m} \times\left(\mathbf{H}_{\mathrm{eff}}+\mathbf{m}_{t}\right) \\
\rho \mathbf{u}_{t t}-\operatorname{div}\left(\mathcal{S}(\mathbf{u})+\frac{1}{2} \mathscr{L}(\mathbf{m})\right) & =0,
\end{aligned}
$$

with associated initial and boundary conditions

$$
\begin{aligned}
\mathbf{u}(\cdot, 0) & =\mathbf{u}_{0}, \\
\mathbf{u}_{t}(\cdot, 0) & =\mathbf{u}_{1}, \\
\mathbf{m}(\cdot, 0) & =\mathbf{m}_{0}, \\
\left|\mathbf{m}_{0}\right| & =1 \\
\mathbf{u} & =0, \\
\mathbf{m}(x, t) & =\mathbf{m}(x+2 \pi, t) \\
& \text { in } \Omega, \\
& \text { on } \Sigma:=\partial \Omega \times(0, T) .
\end{aligned}
$$

The effective field is given by

$$
\mathbf{H}_{\mathrm{eff}}=a \Lambda^{2 \alpha} \mathbf{m}+\boldsymbol{\ell}(\mathbf{m}, \mathbf{u}) \text {, }
$$

where $\Lambda=(-\Delta)^{1 / 2}$ denotes the square root of the Laplacian which can be defined via Fourier transformation [7]. In this paper we are interested in the case $\alpha \in(1 / 2,1)$. For the vector $\mathbf{u}$, we assume that $\mathbf{u}=(0,0, \omega)$ and we keep the three components of the vector $\mathbf{m}=\left(m_{1}, m_{2}, m_{3}\right)$.
It is a common practice (see [5]) to replace the first equation of system (7) by the quasilinear parabolic equation (Ginzburg-Landau type equation):

$$
\mathbf{m}_{t}^{\varepsilon}+\gamma^{-1} \mathbf{m}_{t}^{\varepsilon} \times \mathbf{m}^{\varepsilon}=-\mathbf{H}_{\mathrm{eff}}^{\varepsilon}-\frac{\left|\mathbf{m}^{\varepsilon}\right|^{2}-1}{\varepsilon} \mathbf{m}^{\varepsilon} .
$$

Here $\varepsilon$ is a positive parameter and $\mathbf{m}^{\varepsilon}: \Omega \rightarrow \mathbb{R}^{3} \cdot \varepsilon$ penalization in (11) replaces the magnitude constraint $|\mathbf{m}|=$ 1.

Throughout, we make use of the following notation. For $\Omega$, an open bounded domain of $\mathbb{R}^{3}$, we denote by $\mathbf{L}^{p}(\Omega)=$ $\left(L^{p}(\Omega)\right)^{3}$ and $\mathbf{H}^{1}(\Omega)=\left(H^{1}(\Omega)\right)^{3}$ the classical Hilbert spaces equipped with the usual norm denoted by $\|\cdot\|_{L^{p}(\Omega)}$ and $\| \cdot$ $\|_{\mathbf{H}^{1}(\Omega)}$ (in general, the product functional spaces $(X)^{3}$ are all simplified to $\mathbf{X})$. For all $s>0, W^{s, p}$ denotes the usual Sobolev space consisting of all $f$ such that

$$
\|f\|_{W^{s, p}}:=\left\|\mathscr{F}^{-1}\left(1+|\cdot|^{2}\right)^{s / 2}(\mathscr{F} f)(\cdot)\right\|_{L^{p}}<\infty,
$$

where $\mathscr{F}$ denotes the Fourier transform and $\mathscr{F}^{-1}$ its inverse. Let $\dot{W}^{s, p}$ denote the corresponding homogeneous Sobolev space. When $p=2, W^{s, p}$ corresponds to the usual Sobolev space $H^{s}$.

Now we give a definition of the solution in the weak sense of problem (7)-(8)-(9).

Definition 1. Let $\mathbf{m}_{0} \in \mathbf{H}^{\alpha}(\Omega),\left|\mathbf{m}_{0}\right|=1$ a.e., $\omega_{0} \in H_{0}^{1}(\Omega)$, and $\omega_{1} \in L^{2}(\Omega)$. One says that the pair $(\mathbf{m}, \omega)$ is a weak solution of problem (7)-(8)-(9) if

(i) for all $T>0, \mathbf{m} \in L^{\infty}\left(0, T ; \mathbf{H}^{\alpha}(\Omega)\right), \mathbf{m}_{t} \in$ $L^{2}\left(0, T ; \mathbf{L}^{2}(\Omega)\right),|\mathbf{m}|=1$ a.e., $\omega \in L^{2}\left(0, T ; H_{0}^{1}(\Omega)\right)$, and $\omega_{t} \in L^{2}\left(0, T ; L^{2}(\Omega)\right)$;

(ii) for all $\varphi \in L^{2}\left(0, T ; \mathbf{H}^{\alpha}(\Omega)\right)$ and $\psi \in H_{0}^{1}(Q)$ one has

$$
\begin{aligned}
& \gamma^{-1} \int_{Q} \mathbf{m}_{t} \cdot \boldsymbol{\varphi} d x d t+\int_{Q}\left(\mathbf{m} \times \mathbf{m}_{t}\right) \cdot \boldsymbol{\varphi} d x d t \\
& \quad=a \int_{Q} \Lambda^{\alpha} \mathbf{m} \cdot \Lambda^{\alpha}(\mathbf{m} \times \boldsymbol{\varphi}) d x d t \\
& \quad+\int_{Q}(\boldsymbol{\ell}(\mathbf{m}, \omega) \times \mathbf{m}) \cdot \boldsymbol{\varphi} d x d t \\
& -\rho \int_{Q} \omega_{t} \psi_{t} d x d t+\int_{Q} \omega_{x} \psi_{x} d x d t \\
& \quad+\lambda \int_{Q} m_{1} m_{3} \psi_{x} d x d t=0 ;
\end{aligned}
$$

(iii) $\mathbf{m}(0, x)=\mathbf{m}_{0}(x)$ and $\omega(0, x)=\omega_{0}(x)$ in the trace sense;

(iv) for all $T>0$ we have

$$
\begin{aligned}
& \frac{a}{2} \int_{\Omega}\left|\Lambda^{\alpha} \mathbf{m}(T)\right|^{2} d x+\int_{Q}\left|\mathbf{m}_{t}\right|^{2} d x d t \\
& \quad+\frac{\rho}{2} \int_{\Omega}\left|\omega_{t}(T)\right|^{2} d x+\frac{1}{4} \int_{\Omega}\left|\omega_{x}(T)\right|^{2} d x
\end{aligned}
$$




$$
\begin{aligned}
\leq & \frac{a}{2} \int_{\Omega}\left|\Lambda^{\alpha} \mathbf{m}_{0}\right|^{2} d x+\frac{\rho}{2} \int_{\Omega}\left|\omega_{1}\right|^{2} d x \\
& +\frac{1}{4} \int_{\Omega}\left|\omega_{x}(0)\right|^{2} d x+C(\Omega, \lambda),
\end{aligned}
$$

where $C(\Omega, \lambda)$ is a positive constant which depends only on $\Omega$ and $\lambda$.

The main result of this paper is the following.

Theorem 2. Let $\alpha \in(1 / 2,1), \mathbf{m}_{0} \in \mathbf{H}^{\alpha}(\Omega)$ such that $\left|\mathbf{m}_{0}\right|=1$ a.e., $\omega_{0} \in H_{0}^{1}(\Omega)$, and $\omega_{1} \in L^{2}(\Omega)$. Then there exists at least a weak solution for problem (7)-(8)-(9) in the sense of Definition 1.

The proof of Theorem 2 will be given in Section 4 .

\section{Some Technical Lemmas}

In this section we present some lemmas which will be used in the rest of the paper. We start by recalling the following lemma due to Simon (see [8]).

Lemma 3. Assume $A, B$, and $C$ are three Banach spaces and satisfy $A \subset B \subset C$ with compact embedding $A \hookrightarrow B$. Let $\Theta$ be bounded in $L^{\infty}(0, T ; A)$ and $\Theta_{t}:=\left\{f_{t} ; f \in \Theta\right\}$ be bounded in $L^{p}(0, T ; C), p>1$. Then $\Theta$ is relatively compact in $C([0, T] ; B)$.

There is another lemma whose proof can be found in [[9], page 12].

Lemma 4. Let $\Theta$ be a bounded open set of $\mathbb{R}_{x}^{d} \times \mathbb{R}_{t}, h_{n}$ and $h$ in $L^{q}(\Theta), 1<q<\infty$ such that $\left\|h_{n}\right\|_{L^{q}(\Theta)} \leq C, h_{n} \rightarrow h$ a.e. in $\Theta$; then $h_{n} \rightarrow h$ weakly in $L^{q}(\Theta)$.

The following lemma will ensure a compact embedding for the space $W^{s, p}$.

Lemma 5. Let $\Theta$ be a bounded open set of $\mathbb{R}^{d}$, which is uniform Lipschitz. Let $s \in[0,1[, p>1, d \geq 1$. If $s p<d$ then the injection of $W^{s, p}(\Theta)$ in $L^{k}(\Theta)$ is compact, for any $k<d p /(d-$ $s p)$.

The proof can be found in [[10], Theorem 4.54., p 216]. We give now a lemma that will play a very important role in the convergence of approximate solutions (see [11-13] for a proof).

Lemma 6 (commutator estimates). Suppose that $s>0$ and $p \in(1,+\infty)$. If $f, g \in \mathcal{S}$ (the Schwartz class) then

$$
\begin{aligned}
& \left\|\Lambda^{s}(f g)-f \Lambda^{s} g\right\|_{L^{p}} \\
& \quad \leq C\left(\|\nabla f\|_{L^{p_{1}}}\|g\|_{\dot{W}^{s-1, p_{2}}}+\|f\|_{\dot{W}^{s, p_{3}}}\|g\|_{L^{p_{4}}}\right), \\
& \left\|\Lambda^{s}(f g)\right\|_{L^{p}} \leq C\left(\|f\|_{L^{p_{1}}}\|g\|_{\dot{W}^{s, p_{2}}}+\|f\|_{\dot{W}^{s, p_{3}}}\|g\|_{L^{p_{4}}}\right)
\end{aligned}
$$

with $p_{2}, p_{3} \in(1,+\infty)$ such that $1 / p=1 / p_{1}+1 / p_{2}=1 / p_{3}+$ $1 / p_{4}$.
Here is another lemma which can be viewed as a result of the Hardy-Littlewood-Sobolev theorem of fractional integration; see [7] for a detailed proof.

Lemma 7. Suppose that $p>q>1$ and $1 / p+s=1 / q$. Assume that $f \in L^{q}$; then $\Lambda^{-s} f \in L^{p}$ and there is a constant $C>0$ such that

$$
\|f\|_{\dot{W}^{-s, p}}:=\left\|\Lambda^{-s} f\right\|_{L^{p}} \leq C\|f\|_{L^{q}} .
$$

We finish this section with the following result (the proof can be found in [2]).

Lemma 8. If $f$ and $g$ belong to $H_{\text {per }}^{2 \alpha}(\Omega):=\{f \in$ $\left.L^{2}(\Omega) / \Lambda^{2 \alpha} f \in L^{2}(\Omega)\right\}$, then

$$
\int_{\Omega} \Lambda^{2 \alpha} f \cdot g d x=\int_{\Omega} \Lambda^{\alpha} f \cdot \Lambda^{\alpha} g d x
$$

\section{Proof of Theorem 2}

Our goal is to show global existence of weak solutions for the fractional problem (7)-(8)-(9).

4.1. The Penalty Problem. Let $\varepsilon>0$ be a fixed parameter. We construct approximated solutions $\mathbf{m}^{\varepsilon}$ converging, as $\varepsilon \rightarrow 0$, to a solution $\mathbf{m}$ of the problem. System (7) is reduced to the following problem:

$$
\begin{aligned}
& \gamma^{-1} \mathbf{m}_{t}^{\varepsilon} \times \mathbf{m}^{\varepsilon}+\mathbf{m}_{t}^{\varepsilon}+a \Lambda^{2 \alpha} \mathbf{m}^{\varepsilon}+\boldsymbol{\ell}\left(\mathbf{m}^{\varepsilon}, \omega^{\varepsilon}\right) \\
& +\frac{\left|\mathbf{m}^{\varepsilon}\right|^{2}-1}{\varepsilon} \mathbf{m}^{\varepsilon}=0 \\
& \rho \omega_{t t}^{\varepsilon}-\omega_{x x}^{\varepsilon}-\lambda\left(m_{1}^{\varepsilon} m_{3}^{\varepsilon}\right)_{x}=0
\end{aligned}
$$

in $Q=\Omega \times(0, T)$, where the vector $\boldsymbol{\ell}(\mathbf{m}, \omega)$ is given by $\boldsymbol{\ell}(\mathbf{m}, \omega)=\left(\lambda m_{3} \omega_{x}, 0, \lambda m_{1} \omega_{x}\right), \lambda_{1}=\lambda_{2}=0, \lambda_{3}=\lambda$, and $\sigma_{1313}=1$.

System (19) is supplemented with initial and boundary conditions

$$
\begin{gathered}
\omega^{\varepsilon}(\cdot, 0)=\omega_{0}, \\
\omega_{t}^{\varepsilon}(\cdot, 0)=\omega_{1}, \\
\mathbf{m}^{\varepsilon}(\cdot, 0)=\mathbf{m}_{0}, \\
\left|\mathbf{m}_{0}\right|=1
\end{gathered}
$$

$$
\begin{aligned}
\omega^{\varepsilon} & =0, \\
\mathbf{m}^{\varepsilon}(x, t) & =\mathbf{m}^{\varepsilon}(x+2 \pi, t)
\end{aligned}
$$


We apply Faedo-Galerkin method: let $\left\{f_{i}\right\}_{i \in \mathbb{N}}$ be an orthonormal basis of $L^{2}(\Omega)$ consisting of all the eigenfunctions for the operator $\Lambda^{2 \alpha}$ (the existence of such a basis can be proved as in [14], Ch. II),

$$
\begin{aligned}
\Lambda^{2 \alpha} f_{i} & =\alpha_{i} f_{i}, \quad i=1,2, \ldots, \\
f_{i}(0) & =f_{i}(2 \pi),
\end{aligned}
$$

and let $\left\{g_{i}\right\}_{i \in \mathbb{N}}$ be an orthonormal basis of $L^{2}(\Omega)$ consisting of all the eigenfunctions for the operator $-\Delta$ :

$$
\begin{aligned}
-\Delta g_{i} & =\beta_{i} g_{i}, \quad i=1,2, \ldots, \\
g_{i} & =0 \quad \text { on } \partial \Omega .
\end{aligned}
$$

We then consider the following problem in $Q=\Omega \times(0, T)$ :

$$
\begin{aligned}
& \gamma^{-1} \mathbf{m}_{t}^{\varepsilon, N} \times \mathbf{m}^{\varepsilon, N}+\mathbf{m}_{t}^{\varepsilon, N}+a \Lambda^{2 \alpha} \mathbf{m}^{\varepsilon, N}+\boldsymbol{\ell}\left(\mathbf{m}^{\varepsilon, N}, \omega^{\varepsilon, N}\right) \\
& +\frac{\left|\mathbf{m}^{\varepsilon, N}\right|^{2}-1}{\varepsilon} \mathbf{m}^{\varepsilon, N}=0 \\
& \rho \omega_{t t}^{\varepsilon, N}-\omega_{x x}^{\varepsilon, N}-\lambda\left(m_{1}^{\varepsilon, N} m_{3}^{\varepsilon, N}\right)_{x}=0
\end{aligned}
$$

with initial and boundary conditions,

$$
\begin{aligned}
\omega^{\varepsilon, N}(\cdot, 0) & =\omega^{N}(\cdot, 0), \\
\omega_{t}^{\varepsilon, N}(\cdot, 0) & =\omega_{t}^{N}(\cdot, 0), \\
\mathbf{m}^{\varepsilon, N}(\cdot, 0) & =\mathbf{m}^{N}(\cdot, 0), \\
\omega^{\varepsilon, N} & =0 \\
\mathbf{m}^{\varepsilon, N}(x, t) & =\mathbf{m}^{\varepsilon, N}(x+2 \pi, t) \\
\int_{\Omega} \omega^{N}(x, 0) g_{i}(x) d x & =\int_{\Omega} \omega_{0}(x) g_{i}(x) d x, \\
\int_{\Omega} \omega_{t}^{N}(x, 0) g_{i}(x) d x & =\int_{\Omega} \omega_{1}(x) g_{i}(x) d x \\
\int_{\Omega} \mathbf{m}^{N}(x, 0) f_{i}(x) d x & =\int_{\Omega} \mathbf{m}_{0}(x) f_{i}(x) d x .
\end{aligned}
$$

We are looking for approximate solutions $\left(\mathbf{m}^{\varepsilon, N}, \omega^{\varepsilon, N}\right)$ to (23) under the form

$$
\begin{aligned}
\mathbf{m}^{\varepsilon, N} & =\sum_{i=1}^{N} \mathbf{a}_{i}(t) f_{i}(x), \\
\omega^{\varepsilon, N} & =\sum_{i=1}^{N} b_{i}(t) g_{i}(x) .
\end{aligned}
$$

If we multiply each scalar equation of the first equation of (23) by $f_{i}$ and the second by $g_{i}$ and integrate in $\Omega$ we get to a system of ordinary differential equations in the unknown $\left(\mathbf{a}_{i}(t), b_{i}(t)\right), i=1,2, \ldots, N$. We observe that we can write the first equation in the form

$$
\begin{aligned}
- & a \Lambda^{2 \alpha} \mathbf{m}^{\varepsilon, N}-\boldsymbol{\ell}\left(\mathbf{m}^{\varepsilon, N}, \omega^{\varepsilon, N}\right)-\frac{\left|\mathbf{m}^{\varepsilon, N}\right|^{2}-1}{\varepsilon} \mathbf{m}^{\varepsilon, N} \\
= & \mathbb{A}\left(\mathbf{m}^{\varepsilon, N}\right) \mathbf{m}_{t}^{\varepsilon, N}
\end{aligned}
$$

with

$$
\mathbb{A}=\left(\begin{array}{ccc}
1 & \gamma^{-1} m_{3}^{\varepsilon, N} & -\gamma^{-1} m_{2}^{\varepsilon, N} \\
-\gamma^{-1} m_{3}^{\varepsilon, N} & 1 & \gamma^{-1} m_{1}^{\varepsilon, N} \\
\gamma^{-1} m_{2}^{\varepsilon, N} & -\gamma^{-1} m_{1}^{\varepsilon, N} & 1
\end{array}\right) .
$$

It is clear that the matrix $\mathbb{A}$ is invertible which implies the system of first-order ordinary differential equations is Lipschitz locally; then there exists a local solution to the problem that we can extend on $[0, T]$ using a priori estimates. For this, we multiply the first equation of (23) by $\mathbf{m}_{t}^{\varepsilon, N}$ and the second by $\omega_{t}^{\varepsilon, N}$; integrating in $\Omega$, we obtain (by using Lemma 8)

$$
\begin{gathered}
\int_{\Omega}\left|\mathbf{m}_{t}^{\varepsilon, N}\right|^{2} d x+\frac{a}{2} \frac{d}{d t} \int_{\Omega}\left|\Lambda^{\alpha} \mathbf{m}^{\varepsilon, N}\right|^{2} d x \\
+\lambda \int_{\Omega}\left(m_{1}^{\varepsilon, N} m_{3}^{\varepsilon, N}\right)_{t} \omega_{x}^{\varepsilon, N} d x \\
+\frac{1}{4 \varepsilon} \frac{d}{d t} \int_{\Omega}\left(\left|\mathbf{m}^{\varepsilon, N}\right|^{2}-1\right)^{2} d x=0 \\
\frac{\rho}{2} \frac{d}{d t} \int_{\Omega}\left|\omega_{t}^{\varepsilon, N}\right|^{2} d x+\frac{1}{2} \frac{d}{d t} \int_{\Omega}\left|\omega_{x}^{\varepsilon, N}\right|^{2} d x \\
-\lambda \int_{\Omega}\left(m_{1}^{\varepsilon, N} m_{3}^{\varepsilon, N}\right)_{x} \omega_{t}^{\varepsilon, N} d x=0 .
\end{gathered}
$$

We find after summing

$$
\begin{aligned}
& \int_{\Omega}\left|\mathbf{m}_{t}^{\varepsilon, N}\right|^{2} d x+\frac{a}{2} \frac{d}{d t} \int_{\Omega}\left|\Lambda^{\alpha} \mathbf{m}^{\varepsilon, N}\right|^{2} d x \\
& +\frac{1}{4 \varepsilon} \frac{d}{d t} \int_{\Omega}\left(\left|\mathbf{m}^{\varepsilon, N}\right|^{2}-1\right)^{2} d x+\frac{\rho}{2} \frac{d}{d t} \int_{\Omega}\left|\omega_{t}^{\varepsilon, N}\right|^{2} d x \\
& \quad+\frac{1}{2} \frac{d}{d t} \int_{\Omega}\left|\omega_{x}^{\varepsilon, N}\right|^{2} d x \\
& \quad+\lambda \int_{\Omega}\left(\left(m_{1}^{\varepsilon, N} m_{3}^{\varepsilon, N}\right)_{t} \omega_{x}^{\varepsilon, N}-\left(m_{1}^{\varepsilon, N} m_{3}^{\varepsilon, N}\right)_{x} \omega_{t}^{\varepsilon, N}\right) d x
\end{aligned}
$$$$
=0 \text {. }
$$ 
Abstract and Applied Analysis

5

On the other hand

$$
\begin{aligned}
\frac{d}{d t} \int_{\Omega} m_{1}^{\varepsilon, N} m_{3}^{\varepsilon, N} \omega_{x}^{\varepsilon, N} d x \\
=\int_{\Omega}\left(m_{1}^{\varepsilon, N} m_{3}^{\varepsilon, N}\right)_{t} \omega_{x}^{\varepsilon, N} d x+\int_{\Omega} m_{1}^{\varepsilon, N} m_{3}^{\varepsilon, N} \omega_{x, t}^{\varepsilon, N} d x \\
=\int_{\Omega}\left(m_{1}^{\varepsilon, N} m_{3}^{\varepsilon, N}\right)_{t} \omega_{x}^{\varepsilon, N} d x \\
\quad-\int_{\Omega}\left(m_{1}^{\varepsilon, N} m_{3}^{\varepsilon, N}\right)_{x} \omega_{t}^{\varepsilon, N} d x \\
=\int_{\Omega}\left(\left(m_{1}^{\varepsilon, N} m_{3}^{\varepsilon, N}\right)_{t} \omega_{x}^{\varepsilon, N}-\left(m_{1}^{\varepsilon, N} m_{3}^{\varepsilon, N}\right)_{x} \omega_{t}^{\varepsilon, N}\right) d x
\end{aligned}
$$

Now integrating in time,

$$
\begin{aligned}
\int_{Q}\left|\mathbf{m}_{t}^{\varepsilon, N}\right|^{2} d x+\frac{a}{2} \int_{\Omega}\left|\Lambda^{\alpha} \mathbf{m}^{\varepsilon, N}(T)\right|^{2} d x \\
+\frac{1}{4 \varepsilon} \int_{\Omega}\left(\left|\mathbf{m}^{\varepsilon, N}(T)\right|^{2}-1\right)^{2} d x \\
\quad+\frac{\rho}{2} \int_{\Omega}\left|\omega_{t}^{\varepsilon, N}(T)\right|^{2} d x+\frac{1}{2} \int_{\Omega}\left|\omega_{x}^{\varepsilon, N}(T)\right|^{2} d x \\
\quad+\lambda \int_{\Omega}\left(m_{1}^{\varepsilon, N} m_{3}^{\varepsilon, N} \omega_{x}^{\varepsilon, N}\right)(T) d x \\
=\frac{a}{2} \int_{\Omega}\left|\Lambda^{\alpha} \mathbf{m}^{N}(0)\right|^{2} d x \\
\quad+\frac{1}{4 \varepsilon} \int_{\Omega}\left(\left|\mathbf{m}^{N}(0)\right|^{2}-1\right)^{2} d x \\
\quad+\frac{\rho}{2} \int_{\Omega}\left|\omega_{t}^{N}(0)\right|^{2} d x+\frac{1}{2} \int_{\Omega}\left|\omega_{x}^{N}(0)\right|^{2} d x \\
\quad+\lambda \int_{\Omega}\left(m_{1}^{N} m_{3}^{N} \omega_{x}^{N}\right)(0) d x .
\end{aligned}
$$

Omitting superscripts, we obtain for all $b>0$,

$$
\begin{aligned}
\left|\int_{\Omega} m_{1} m_{3} \omega_{x} d x\right| \leq & \int_{\Omega}\left(\frac{1}{2 b}\left(m_{1} m_{3}\right)^{2}+\frac{b}{2} \omega_{x}^{2}\right) d x \\
\leq & \frac{1}{2 b} \int_{\Omega}|\mathbf{m}|^{4} d x+\frac{b}{2} \int_{\Omega} \omega_{x}^{2} d x \\
= & \frac{1}{2 b} \int_{\Omega}\left(|\mathbf{m}|^{2}-1+1\right)^{2} d x \\
& +\frac{b}{2} \int_{\Omega} \omega_{x}^{2} d x \\
\leq & \frac{1}{b} \int_{\Omega}\left(|\mathbf{m}|^{2}-1\right)^{2} d x+\frac{2 \pi}{b} \\
& +\frac{b}{2} \int_{\Omega} \omega_{x}^{2} d x \\
= & \frac{1}{b} \int_{\Omega}\left(|\mathbf{m}|^{2}-1\right)^{2} d x+\frac{2 \pi}{b} \\
& +\frac{b}{2}\left\|\omega_{x}\right\|_{L^{2}(\Omega)}^{2}
\end{aligned}
$$




$$
\begin{aligned}
& \leq 2 \int_{\Omega}\left|\omega_{x}^{N}(0)-\omega_{x}^{0}\right|^{2} d x+2 \int_{\Omega}\left|\omega_{x}^{0}\right|^{2} d x \\
& \leq 2\left\|\omega^{N}(0)-\omega_{0}\right\|_{H_{0}^{1}(\Omega)}^{2}+2\left\|\omega_{0}\right\|_{H_{0}^{1}(\Omega)}^{2} \leq C,
\end{aligned}
$$

$$
\omega^{\varepsilon, N} \longrightarrow \omega^{\varepsilon}
$$

strongly in $L^{2}(Q)$.

thanks to the strong convergence $\omega^{N}(\cdot, 0) \rightarrow \omega_{0}$ in $H_{0}^{1}(\Omega)$. For the other term $\left(\omega_{t}^{N}(0)\right)$, the estimate can be carried out in an analogous way using the strong convergence $\omega_{t}^{N}(\cdot, 0) \rightarrow \omega_{1}$ in $L^{2}(\Omega)$. Moreover, noting that (for a constant $C$ independent of $\varepsilon$ and $N$ )

$$
\begin{aligned}
\int_{\Omega}\left|\mathbf{m}^{\varepsilon, N}\right|^{2} d x & =\int_{\Omega}\left(\left|\mathbf{m}^{\varepsilon, N}\right|^{2}-1+1\right) d x \\
& \leq \frac{1}{2} \int_{\Omega}\left(\left|\mathbf{m}^{\varepsilon, N}\right|^{2}-1\right)^{2} d x+C,
\end{aligned}
$$

therefore, for fixed $\varepsilon>0$, we have

$$
\begin{gathered}
\left(\mathbf{m}^{\varepsilon, N}\right)_{N} \text { is bounded in } L^{\infty}\left(0, T ; \mathbf{H}^{\alpha}(\Omega)\right), \\
\left(\mathbf{m}_{t}^{\varepsilon, N}\right)_{N} \text { is bounded in } L^{2}\left(0, T ; \mathbf{L}^{2}(\Omega)\right), \\
\left(\left|\mathbf{m}^{\varepsilon, N}\right|^{2}-1\right)_{N} \text { is bounded in } L^{\infty}\left(0, T ; L^{2}(\Omega)\right), \\
\left(\omega^{\varepsilon, N}\right)_{N} \text { is bounded in } L^{2}\left(0, T ; H_{0}^{1}(\Omega)\right), \\
\left(\omega_{t}^{\varepsilon, N}\right)_{N} \text { is bounded in } L^{2}\left(0, T ; L^{2}(\Omega)\right) .
\end{gathered}
$$

Note that (37) is due to the Poincaré lemma. Now, from classical compactness results there exist two subsequences which we still denote by $\left(\mathbf{m}^{\varepsilon, N}\right)$ and $\left(\omega^{\varepsilon, N}\right)$ such that for fixed $\varepsilon>0$ and for any $1<p<\infty$

$$
\begin{aligned}
& \mathbf{m}^{\varepsilon, N} \rightarrow \mathbf{m}^{\varepsilon} \\
& \text { weakly in } L^{p}\left(0, T ; \mathbf{H}^{\alpha}(\Omega)\right) \text {, } \\
& \mathbf{m}^{\varepsilon, N} \longrightarrow \mathbf{m}^{\varepsilon} \\
& \text { strongly in } C\left([0, T], \mathbf{H}^{\beta}(\Omega)\right) \text {, a.e. for } 0 \leq \beta<\alpha \\
& \mathbf{m}_{t}^{\varepsilon, N} \rightarrow \mathbf{m}_{t}^{\varepsilon} \\
& \text { weakly in } L^{2}\left(0, T ; \mathbf{L}^{2}(\Omega)\right) \text {, } \\
& \left|\mathbf{m}^{\varepsilon, N}\right|^{2}-1-\zeta \\
& \omega^{\varepsilon, N} \rightarrow \omega^{\varepsilon} \\
& \text { weakly in } L^{p}\left(0, T ; L^{2}(\Omega)\right) \text {, } \\
& \text { weakly in } L^{2}\left(0, T ; H_{0}^{1}(\Omega)\right) \text {, } \\
& \omega_{t}^{\varepsilon, N} \rightarrow \omega_{t}^{\varepsilon} \\
& \text { weakly in } L^{2}\left(0, T ; L^{2}(\Omega)\right) \text {, }
\end{aligned}
$$

Convergence (38) is due to Lemma 3 and thanks to Lemma 4 it can be shown that $\zeta=\left|\mathbf{m}^{\varepsilon}\right|^{2}-1$. Moreover from the Sobolev embedding (Lemma 5) $H^{\alpha}(Q) \hookrightarrow L^{4}(Q)$, the further compactness result follows:

$$
m_{i}^{\varepsilon, N} m_{j}^{\varepsilon, N} \longrightarrow m_{i}^{\varepsilon} m_{j}^{\varepsilon} \quad \text { strongly in } L^{2}(Q)
$$

The above estimates allow us to pass to the limit as $N$ goes to infinity and to get the desired result. Indeed consider the variational formulation of (23):

$$
\begin{aligned}
& \gamma^{-1} \int_{Q} \mathbf{m}_{t}^{\varepsilon, N} \times \mathbf{m}^{\varepsilon, N} \cdot \phi d x d t+\int_{Q} \mathbf{m}_{t}^{\varepsilon, N} \cdot \phi d x d t \\
& +a \int_{Q} \Lambda^{\alpha} \mathbf{m}^{\varepsilon, N} \cdot \Lambda^{\alpha} \phi d x d t \\
& +\int_{Q} \boldsymbol{\ell}\left(\mathbf{m}^{\varepsilon, N}, \omega^{\varepsilon, N}\right) \cdot \phi d x d t \\
& +\int_{Q} \frac{\left|\mathbf{m}^{\varepsilon, N}\right|^{2}-1}{\varepsilon} \mathbf{m}^{\varepsilon, N} \cdot \phi d x d t=0 \\
& -\rho \int_{Q} \omega_{t}^{\varepsilon, N} \psi_{t} d x d t+\int_{Q} \omega_{x}^{\varepsilon, N} \psi_{x} d x d t \\
& +\lambda \int_{Q} m_{1}^{\varepsilon, N} m_{3}^{\varepsilon, N} \psi_{x} d x d t=0,
\end{aligned}
$$

for any $\phi \in L^{2}\left(0, T ; \mathbf{H}^{\alpha}(\Omega)\right)$ and $\psi \in H_{0}^{1}(Q)$. Taking $N \rightarrow \infty$ in (40), we find

$$
\begin{aligned}
& \gamma^{-1} \int_{Q} \mathbf{m}_{t}^{\varepsilon} \times \mathbf{m}^{\varepsilon} \cdot \phi d x d t+\int_{Q} \mathbf{m}_{t}^{\varepsilon} \cdot \phi d x d t \\
& +a \int_{Q} \Lambda^{\alpha} \mathbf{m}^{\varepsilon} \cdot \Lambda^{\alpha} \phi d x d t+\int_{Q} \boldsymbol{\ell}\left(\mathbf{m}^{\varepsilon}, \omega^{\varepsilon}\right) \cdot \phi d x d t \\
& \quad+\int_{Q} \frac{\left|\mathbf{m}^{\varepsilon}\right|^{2}-1}{\varepsilon} \mathbf{m}^{\varepsilon} \cdot \phi d x d t=0, \\
& -\rho \int_{Q} \omega_{t}^{\varepsilon} \psi_{t} d x d t+\int_{Q} \omega_{x}^{\varepsilon} \psi_{x} d x d t \\
& \quad+\lambda \int_{Q} m_{1}^{\varepsilon} m_{3}^{\varepsilon} \psi_{x} d x d t=0,
\end{aligned}
$$

for any $\phi \in L^{2}\left(0, T ; \mathbf{H}^{\alpha}(\Omega)\right)$ and $\psi \in H_{0}^{1}(Q)$. We proved the following result.

Proposition 9. Given $\mathbf{m}_{0} \in \mathbf{H}^{\alpha}(\Omega)$ such that $\left|\mathbf{m}_{0}\right|=1$ a.e., $\omega_{0} \in H_{0}^{1}(\Omega)$, and $\omega_{1} \in L^{2}(\Omega)$, then there exists a solution $\mathbf{m}^{\varepsilon}$ 
to problem (19) in the sense of distributions. Moreover we have the following energy estimate:

$$
\begin{aligned}
\frac{a}{2} \int_{\Omega} \mid & \left.\Lambda^{\alpha} \mathbf{m}^{\varepsilon}(T)\right|^{2} d x+\int_{Q}\left|\mathbf{m}_{t}^{\varepsilon}(T)\right|^{2} d x d t \\
& +\frac{1}{8 \varepsilon} \int_{\Omega}\left(\left|\mathbf{m}^{\varepsilon}(T)\right|^{2}-1\right)^{2} d x \\
& +\frac{\rho}{2} \int_{\Omega}\left|\omega_{t}^{\varepsilon}(T)\right|^{2} d x+\frac{1}{4} \int_{\Omega}\left|\omega_{x}^{\varepsilon}(T)\right|^{2} d x \\
\leq & \frac{a}{2} \int_{\Omega}\left|\Lambda^{\alpha} \mathbf{m}_{0}\right|^{2} d x+8 \pi \lambda^{2}+\frac{\rho}{2} \int_{\Omega}\left|\omega_{1}\right|^{2} d x \\
& +\frac{1}{4} \int_{\Omega}\left|\omega_{x}(0)\right|^{2} d x .
\end{aligned}
$$

4.2. Convergence of Approximate Solutions. Our aim here is to pass to the limit as $\varepsilon \rightarrow 0$. For this, we will use estimate (42), from which we have the following:

$$
\begin{gathered}
\left(\mathbf{m}^{\varepsilon}\right)_{\varepsilon} \text { is bounded in } L^{\infty}\left(0, T ; \mathbf{H}^{\alpha}(\Omega)\right), \\
\left(\mathbf{m}_{t}^{\varepsilon}\right)_{\varepsilon} \text { is bounded in } L^{2}\left(0, T ; \mathbf{L}^{2}(\Omega)\right), \\
\left(\left|\mathbf{m}^{\varepsilon}\right|^{2}-1\right)_{\varepsilon} \text { is bounded in } L^{\infty}\left(0, T ; L^{2}(\Omega)\right), \\
\left(\omega^{\varepsilon}\right)_{\varepsilon} \text { is bounded in } L^{2}\left(0, T ; H_{0}^{1}(\Omega)\right), \\
\left(\omega_{t}^{\varepsilon}\right)_{\varepsilon} \text { is bounded in } L^{2}\left(0, T ; L^{2}(\Omega)\right) .
\end{gathered}
$$

Then there exist two subsequences which we still denote by $\left(\mathbf{m}^{\varepsilon}\right)$ and $\left(\omega^{\varepsilon}\right)$ such that for any $1<p<\infty$

$$
\begin{aligned}
& \mathbf{m}^{\varepsilon} \rightarrow \mathbf{m} \\
& \mathbf{m}^{\varepsilon} \longrightarrow \mathbf{m}
\end{aligned}
$$

strongly in $C\left([0, T], \mathbf{H}^{\beta}(\Omega)\right)$, a.e. for $0 \leq \beta<\alpha$

$$
\begin{aligned}
\mathbf{m}_{t}^{\varepsilon} & \rightarrow \mathbf{m}_{t} \\
\left|\mathbf{m}^{\varepsilon}\right|^{2}-1 & \longrightarrow 0
\end{aligned}
$$$$
\text { strongly in } L^{p}\left(0, T ; L^{2}(\Omega)\right) \text {, a.e. }
$$

$$
\begin{array}{ll}
\omega^{\varepsilon} \rightarrow \omega & \\
& \text { weakly in } L^{2}\left(0, T ; H_{0}^{1}(\Omega)\right), \\
\omega_{t}^{\varepsilon} \rightarrow \omega_{t} & \text { weakly in } L^{2}\left(0, T ; L^{2}(\Omega)\right), \\
\omega^{\varepsilon} \longrightarrow \omega &
\end{array}
$$$$
\text { strongly in } L^{2}(Q) \text {. }
$$

It can be shown from convergence (44) that $|\mathbf{m}|=1$ a.e.

Now in order to pass to the limit $\varepsilon \rightarrow 0$ in (41), let $\varphi \in$ $\mathbf{C}^{\infty}([0, T] \times \bar{\Omega})$, and let $\phi=\mathbf{m}^{\varepsilon} \times \boldsymbol{\varphi}$. We first recall the identity $(\mathbf{a} \times \mathbf{b}) \cdot(\mathbf{c} \times \mathbf{d})=(\mathbf{a} \cdot \mathbf{c})(\mathbf{b} \cdot \mathbf{d})-(\mathbf{a} \cdot \mathbf{d})(\mathbf{b} \cdot \mathbf{c})$ for all $\mathbf{a}, \mathbf{b}, \mathbf{c}$, and d in $\mathbb{R}^{3}$.

As $\phi$ is in $L^{2}\left(0, T ; \mathbf{H}^{\alpha}(\Omega)\right)$, the following holds:

$$
\begin{aligned}
& \gamma^{-1} \int_{Q}\left(\mathbf{m}_{t}^{\varepsilon} \cdot \mathbf{m}^{\varepsilon}\right)\left(\mathbf{m}^{\varepsilon} \cdot \boldsymbol{\varphi}\right) d x d t \\
& \quad-\gamma^{-1} \int_{Q}\left(\mathbf{m}_{t}^{\varepsilon} \cdot \boldsymbol{\varphi}\right)\left|\mathbf{m}^{\varepsilon}\right|^{2} d x d t \\
& \quad+\int_{Q}\left(\mathbf{m}_{t}^{\varepsilon} \times \mathbf{m}^{\varepsilon}\right) \cdot \boldsymbol{\varphi} d x d t \\
& +a \int_{Q} \Lambda^{\alpha} \mathbf{m}^{\varepsilon} \cdot \Lambda^{\alpha}\left(\mathbf{m}^{\varepsilon} \times \boldsymbol{\varphi}\right) d x d t \\
& \quad+\int_{Q}\left(\boldsymbol{\ell}\left(\mathbf{m}^{\varepsilon}, \omega^{\varepsilon}\right) \times \mathbf{m}^{\varepsilon}\right) \cdot \boldsymbol{\varphi} d x d t=0 \\
& \quad-\rho \int_{Q} \omega_{t}^{\varepsilon} \psi_{t} d x d t+\int_{Q} \omega_{x}^{\varepsilon} \psi_{x} d x d t \\
& \quad+\lambda \int_{Q} m_{1}^{\varepsilon} m_{3}^{\varepsilon} \psi_{x} d x d t=0 .
\end{aligned}
$$

Note that for this choice we have $\Lambda^{\alpha}\left(\mathbf{m}^{\varepsilon} \times \varphi\right) \in \mathbf{L}^{2}(Q)$, indeed applying the multiplicative estimates (16) in Lemma 6 to $\mathbf{m}^{\varepsilon}$ and $\varphi$ (for $s=\alpha, p=2, p_{1}=\infty, p_{2}=2, p_{3}=2$, and $p_{4}=\infty$ ); we find for different constants $C$ independent of $\varepsilon$ :

$$
\begin{aligned}
& \left\|\Lambda^{\alpha}\left(\mathbf{m}^{\varepsilon} \times \boldsymbol{\varphi}\right)\right\|_{\mathbf{L}^{2}(Q)} \\
& \quad \leq C\left(\left\|\mathbf{m}^{\varepsilon}\right\|_{L^{\infty}(Q)}\|\varphi\|_{\dot{H}^{\alpha}(Q)}+\left\|\mathbf{m}^{\varepsilon}\right\|_{\dot{H}^{\alpha}(Q)}\|\varphi\|_{L^{\infty}(Q)}\right) \\
& \quad \leq C ;
\end{aligned}
$$

since $2 \alpha>1$ ( 1 here is the dimension) then $\mathbf{H}^{\alpha}(\Omega) \hookrightarrow \mathbf{L}^{\infty}(\Omega)$ and consequently $\left(\mathbf{m}^{\varepsilon}\right)_{\varepsilon}$ is bounded in $\mathbf{L}^{\infty}(Q)$. have

Taking $\varepsilon \rightarrow 0$ and following the idea introduced in [4] we

$$
\int_{Q}\left(\mathbf{m}_{t}^{\varepsilon} \cdot \mathbf{m}^{\varepsilon}\right)\left(\mathbf{m}^{\varepsilon} \cdot \boldsymbol{\varphi}\right) d x d t
$$

$$
\begin{aligned}
& =\int_{Q}\left(\mathbf{m}_{t}^{\varepsilon} \cdot \mathbf{m}^{\varepsilon}\right)\left(\mathbf{m}^{\varepsilon} \cdot \boldsymbol{\varphi}\right) d x d t \\
& \quad-\int_{Q}\left(\mathbf{m}_{t} \cdot \mathbf{m}\right)(\mathbf{m} \cdot \boldsymbol{\varphi}) d x d t \\
& =\int_{Q}\left(\mathbf{m}_{t}^{\varepsilon} \cdot \mathbf{m}^{\varepsilon}\right)\left(\mathbf{m}^{\varepsilon}-\mathbf{m}\right) \cdot \boldsymbol{\varphi} d x d t \\
& \quad+\int_{Q}\left(\mathbf{m}^{\varepsilon} \cdot\left(\mathbf{m}_{t}^{\varepsilon}-\mathbf{m}_{t}\right)\right)(\mathbf{m} \cdot \boldsymbol{\varphi}) d x d t \\
& \quad+\int_{Q}\left(\mathbf{m}_{t} \cdot\left(\mathbf{m}^{\varepsilon}-\mathbf{m}\right)\right)(\mathbf{m} \cdot \boldsymbol{\varphi}) d x d t \longrightarrow 0
\end{aligned}
$$

$\int_{Q}\left|\mathbf{m}^{\varepsilon}\right|^{2} \mathbf{m}_{t}^{\varepsilon} \cdot \boldsymbol{\varphi} d x d t$ 


$$
\begin{aligned}
& =\int_{Q}\left(\left|\mathbf{m}^{\varepsilon}\right|^{2}-1\right) \mathbf{m}_{t}^{\varepsilon} \cdot \boldsymbol{\varphi} d x d t+\int_{Q} \mathbf{m}_{t}^{\varepsilon} \cdot \boldsymbol{\varphi} d x d t \\
& \longrightarrow \int_{Q} \mathbf{m}_{t} \cdot \boldsymbol{\varphi} d x d t .
\end{aligned}
$$

Now for the fourth term of the first equation, we introduce the commutator (see [4]):

$$
\Gamma_{\varphi}(\mathbf{m}):=\Lambda^{\alpha}(\mathbf{m} \times \varphi)-\varphi \times \Lambda^{\alpha} \mathbf{m}
$$

Let $\mathfrak{I}_{\varepsilon}=\int_{Q} \Lambda^{\alpha} \mathbf{m}^{\varepsilon} \cdot \Lambda^{\alpha}\left(\mathbf{m}^{\varepsilon} \times \boldsymbol{\varphi}\right) d x d t$ and $\mathfrak{I}=\int_{Q} \Lambda^{\alpha} \mathbf{m}$. $\Lambda^{\alpha}(\mathbf{m} \times \varphi) d x d t$. We will show that $\mathfrak{J}_{\varepsilon} \rightarrow \mathfrak{I}$.

First, note that $\Gamma_{\varphi}(\mathbf{m}) \in \mathbf{L}^{2}(Q)$. Indeed, applying (15) for $p_{1}=1 /(1-\alpha), p_{2}=2 /(2 \alpha-1), p_{3}$ and $p_{4}$ in $(2,+\infty)$, we find (for different constants $C$ )

$$
\begin{aligned}
& \left\|\Gamma_{\boldsymbol{\varphi}}(\mathbf{m})\right\|_{\mathbf{L}^{2}(\Omega)} \leq C\left(\|\nabla \boldsymbol{\varphi}\|_{\mathbf{L}^{p_{1}}(\Omega)}\|\mathbf{m}\|_{\dot{\mathbf{W}}^{\alpha-1, p_{2}}(\Omega)}\right. \\
& \left.\quad+\|\varphi\|_{\dot{\mathbf{W}}^{\alpha, p_{3}}(\Omega)}\|\mathbf{m}\|_{\mathbf{L}^{p_{4}}(\Omega)}\right) \\
& \quad \leq C\left(\|\nabla \boldsymbol{\varphi}\|_{\mathbf{L}^{p_{1}(\Omega)}}\|\mathbf{m}\|_{\mathbf{L}^{2}(\Omega)}+\|\boldsymbol{\varphi}\|_{\dot{\mathbf{W}}^{\alpha, p_{3}}(\Omega)}\|\mathbf{m}\|_{\mathbf{L}^{2}(\Omega)}\right) \\
& \quad \leq C\|\mathbf{m}\|_{\mathbf{L}^{2}(\Omega)},
\end{aligned}
$$

thanks to Lemma 7. Once again

$$
\begin{aligned}
& \left\|\Gamma_{\boldsymbol{\varphi}}\left(\mathbf{m}^{\varepsilon}-\mathbf{m}\right)\right\|_{\mathbf{L}^{2}(\Omega)} \\
& \quad \leq C\left(\|\nabla \boldsymbol{\varphi}\|_{\mathbf{L}^{p_{1}}(\Omega)}\left\|\mathbf{m}^{\varepsilon}-\mathbf{m}\right\|_{\dot{\mathbf{W}}^{\alpha-1, p_{2}}(\Omega)}\right. \\
& \left.\quad+\|\varphi\|_{\dot{\mathbf{W}}^{\alpha, p_{3}}(\Omega)}\left\|\mathbf{m}^{\varepsilon}-\mathbf{m}\right\|_{\mathbf{L}^{p_{4}}(\Omega)}\right) \\
& \left\|\Gamma_{\boldsymbol{\varphi}}\left(\mathbf{m}^{\varepsilon}-\mathbf{m}\right)\right\|_{\mathbf{L}^{2}(Q)} \\
& \quad \leq C\left(\|\nabla \boldsymbol{\varphi}\|_{L^{\infty}\left(0, T ; \mathbf{L}^{p_{1}}(\Omega)\right)}\left\|\mathbf{m}^{\varepsilon}-\mathbf{m}\right\|_{\mathbf{L}^{2}(Q)}\right. \\
& \left.\quad+\|\varphi\|_{L^{\infty}\left(0, T ; \dot{\mathbf{W}}^{\alpha, p_{3}}(\Omega)\right)}\left\|\mathbf{m}^{\varepsilon}-\mathbf{m}\right\|_{L^{2}\left(0, T ; \mathbf{H}^{\beta}(\Omega)\right)}\right),
\end{aligned}
$$

where the right-hand side of the last inequality tends to 0 .

Finally, we have

$$
\begin{aligned}
& \left|\mathfrak{\Im}_{\varepsilon}-\mathfrak{I}\right|=\mid \int_{Q} \Lambda^{\alpha} \mathbf{m}^{\varepsilon} \cdot \Gamma_{\varphi}\left(\mathbf{m}^{\varepsilon}\right) d x d t \\
& \quad-\int_{Q} \Lambda^{\alpha} \mathbf{m} \cdot \Gamma_{\varphi}(\mathbf{m}) d x d t \mid \\
& \quad=\mid \int_{Q} \Lambda^{\alpha} \mathbf{m}^{\varepsilon} \cdot \Gamma_{\varphi}\left(\mathbf{m}^{\varepsilon}-\mathbf{m}\right) d x d t \\
& \quad+\int_{Q} \Lambda^{\alpha}\left(\mathbf{m}^{\varepsilon}-\mathbf{m}\right) \cdot \Gamma_{\varphi}(\mathbf{m}) d x d t \mid \\
& \quad \leq \int_{Q}\left|\Lambda^{\alpha} \mathbf{m}^{\varepsilon} \cdot \Gamma_{\varphi}\left(\mathbf{m}^{\varepsilon}-\mathbf{m}\right)\right| d x d t \\
& \quad+\left|\int_{Q} \Lambda^{\alpha}\left(\mathbf{m}^{\varepsilon}-\mathbf{m}\right) \cdot \Gamma_{\varphi}(\mathbf{m}) d x d t\right|
\end{aligned}
$$

$$
\begin{aligned}
& \leq C\left\|\Gamma_{\varphi}\left(\mathbf{m}^{\varepsilon}-\mathbf{m}\right)\right\|_{L^{2}(Q)} \\
& +\left|\int_{Q} \Lambda^{\alpha}\left(\mathbf{m}^{\varepsilon}-\mathbf{m}\right) \cdot \Gamma_{\varphi}(\mathbf{m}) d x d t\right| \longrightarrow 0 .
\end{aligned}
$$

Therefore

$$
\begin{aligned}
& \gamma^{-1} \int_{Q} \mathbf{m}_{t} \cdot \boldsymbol{\varphi} d x d t+\int_{Q}\left(\mathbf{m} \times \mathbf{m}_{t}\right) \cdot \boldsymbol{\varphi} d x d t \\
& \quad=a \int_{Q} \Lambda^{\alpha} \mathbf{m} \cdot \Lambda^{\alpha}(\mathbf{m} \times \boldsymbol{\varphi}) d x d t \\
& \quad+\int_{Q}(\boldsymbol{\ell}(\mathbf{m}, \omega) \times \mathbf{m}) \cdot \boldsymbol{\varphi} d x d t, \\
& -\rho \int_{Q} \omega_{t} \psi_{t} d x d t+\int_{Q} \omega_{x} \psi_{x} d x d t \\
& \quad+\lambda \int_{Q} m_{1} m_{3} \psi_{x} d x d t=0 .
\end{aligned}
$$

This being true for every $\boldsymbol{\varphi} \in \mathbf{C}^{\infty}([0, T] \times \bar{\Omega})$ and by a standard density argument, it is true for any $\varphi$ in $L^{2}\left(0, T ; \mathbf{H}^{\alpha}(\Omega)\right)$. Note that, from (42), one can easily get (14). Hence $(\mathbf{m}, \omega)$ is a solution of problem (7)-(8)-(9) in the sense of Definition 1. The proof of Theorem 2 is complete.

\section{Competing Interests}

The authors declare that there is no conflict of interests regarding the publication of this paper.

\section{Acknowledgments}

The research is supported by the PHC Volubilis Program MA/14/301 "Elaboration et analyse de modèles asymptotiques en micro-magnétisme, magnéto-élasticité et électroélasticité" with joint financial support from the French Ministry of Foreign Affairs and the Moroccan Ministry of Higher Education and Scientific Research.

\section{References}

[1] V. Valente and G. Vergara Caffarelli, "On the dynamics of magneto-elastic interactions: existence of solutions and limit behaviors," Asymptotic Analysis, vol. 51, no. 3-4, pp. 319-333, 2007.

[2] B. Guo and M. Zeng, "Solutions for the fractional LandauLifshitz equation," Journal of Mathematical Analysis and Applications, vol. 361, no. 1, pp. 131-138, 2010.

[3] X. Pu and B. Guo, "Well-posedness for the fractional LandauLifshitz equation without Gilbert damping," Calculus of Variations and Partial Differential Equations, vol. 46, no. 3-4, pp. 441460, 2013.

[4] X. Pu, B. Guo, and J. Zhang, "Global weak solutions to the 1-D fractional Landau-Lifshitz equation," Discrete and Continuous Dynamical Systems Series B, vol. 14, no. 1, pp. 199-207, 2010.

[5] M. Chipot, I. Shafrir, V. Valente, and G. Vergara Caffarelli, "On a hyperbolic-parabolic system arising in magnetoelasticity," 
Journal of Mathematical Analysis and Applications, vol. 352, no. 1, pp. 120-131, 2009.

[6] M. Chipot, I. Shafrir, V. Valente, and G. Vergara Caffarelli, "A nonlocal problem arising in the study of magneto-elastic interactions," Bollettino dell'Unione Matematica Italiana, vol. 9, no. 1, pp. 197-221, 2008.

[7] E. M. Stein, Singular Integrals and Differentiability Properties of Functions, Princeton Mathematical Series, Princeton University Press, Princeton, NJ, USA, 1970.

[8] J. Simon, "Compact sets in the space $L^{p}(O, T ; B)$," Annali di Matematica Pura ed Applicata, vol. 146, pp. 65-96, 1987.

[9] J. L. Lions, Quelques Méthodes de Résolution des Problémes aux Limites Non Linéaires, Dunod, Paris, France, 1969.

[10] F. Demengel and G. Demengel, Functional Spaces for the Theory of Elliptic Partial Differential Equations, Universitext, Springer, London, UK, 2012, Translated from the French Original by R. Erné, EDP Sciences, Les Ulis, France, 2007.

[11] R. R. Coifman and Y. Meyer, "Nonlinear harmonic analysis, operator theory and P.D.E.", in Beijing Lectures in Harmonic Analysis, Annals of Mathematics Studies, pp. 3-45, Princeton University Press, 1986.

[12] T. Kato, "Liapunov functions and monotonicity in the navierstokes equations," in Functional-Analytic Methods for Partial Differential Equations, vol. 1450 of Lecture Notes in Mathematics, pp. 53-63, Springer, Berlin, Germany, 1990.

[13] T. Kato and G. Ponce, "Commutator estimates and the Euler and Navier-Stokes equations," Communications on Pure and Applied Mathematics, vol. 41, no. 7, pp. 891-907, 1988.

[14] R. Temam, Infinite-Dimensional Dynamical Systems in Mechanics and Physics, vol. 68 of Applied Mathematical Sciences, Springer, New York, NY, USA, 1997. 


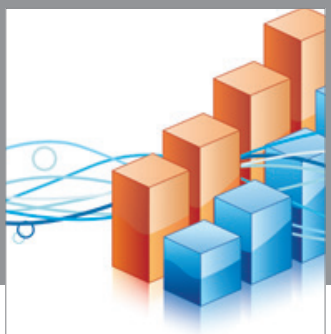

Advances in

Operations Research

vatem alat4

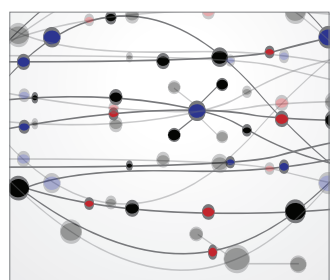

\section{The Scientific} World Journal
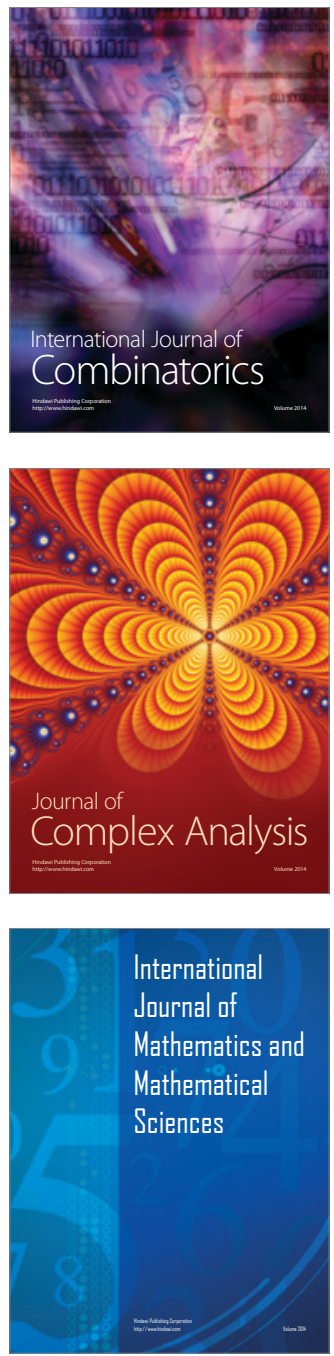
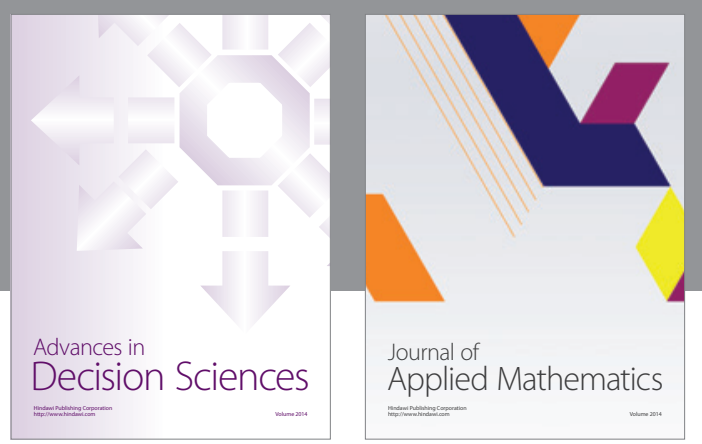

Algebra

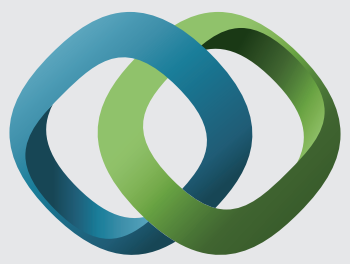

\section{Hindawi}

Submit your manuscripts at

http://www.hindawi.com
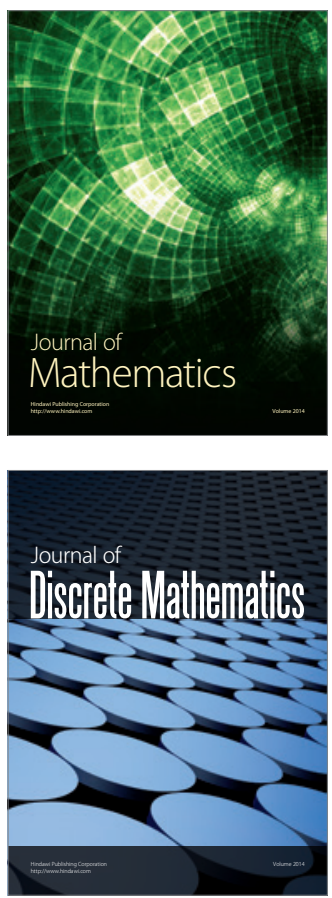

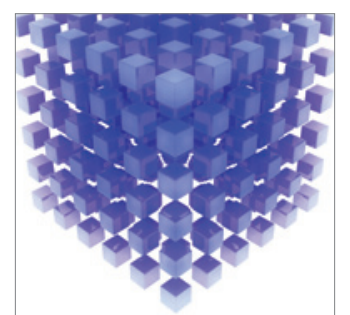

Mathematical Problems in Engineering
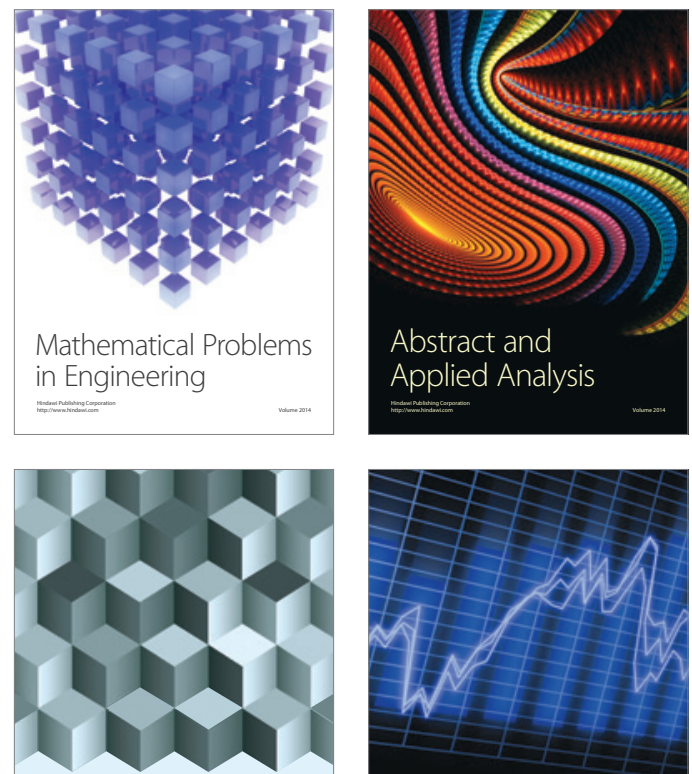

Journal of

Function Spaces

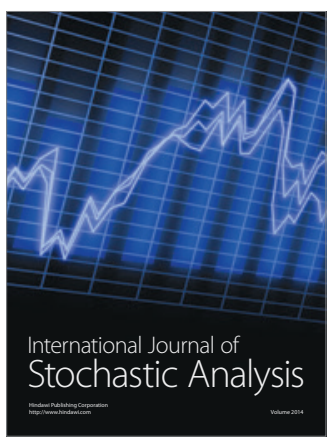

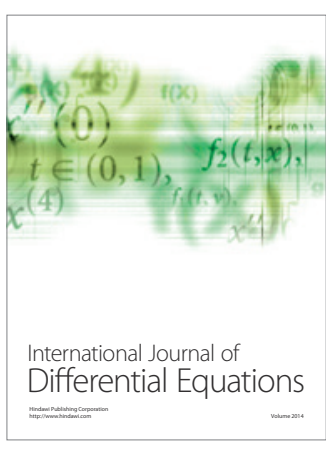
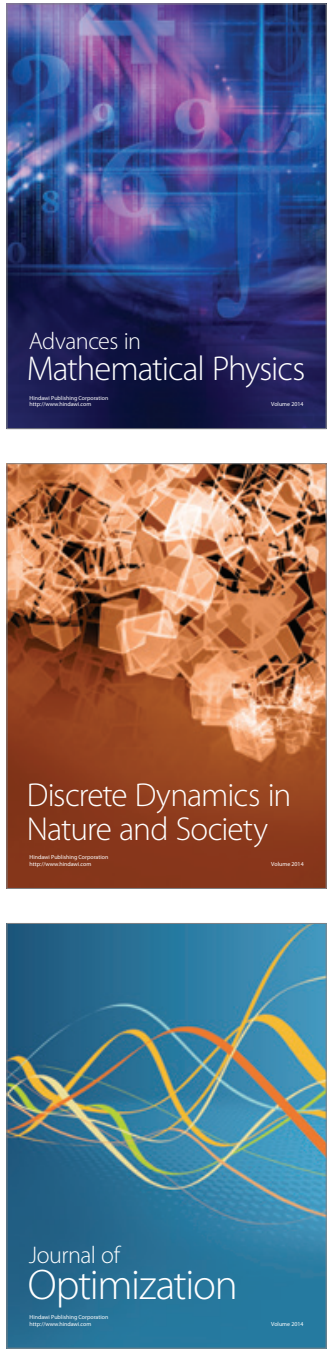